\title{
EXTENDING ISOMETRIC EMBEDDINGS
}

\author{
H. JACOBOWITZ
}

\section{Introduction}

Let $H$ be a submanifold of a Riemannian manifold $U$. Assume $f: H \rightarrow E^{N}$ is an isometric embedding into some Euclidean space. When can $f$ be extended to an isometric embedding of $U$ into the same Euclidean space? In this paper the author considers the local problem of finding conditions on $f$ and $H$ at a point $p \in H$, which guarantee the existence of such an extension of $f$ to an open neighborhood in $U$ containing $p$. When everything is real analytic, these conditions are implicit in the proofs of the Cartan-Janet theorem. However, by focusing on this extension problem we obtain a proof which appears to be simpler and better motivated than those in the literature. The author's main aim is to obtain such extension results even in the nonanalytic case. The approach is basically that of Nash [11]. One inverts a linearized operator and tries to construct the solutions of a nonlinear problem. The present work is based on the author's version [7] of Nash's paper.

To see the geometry involved consider a two-dimensional Riemannian manifold $U$ and the isometric embedding of a curve $H$ on the manifold into three dimensional Euclidean space. In general such an isometric embedding cannot be extended. This is clear, for instance, if $H$ is not a geodesic but its image is. More generally compare the geodesic curvature $\kappa_{g}$ of $H$ at $p$ with the curvature of the space curve which is its image. Let $\boldsymbol{e}$ be the curvature vector at $f(p)$. If an extension exists, then there must exist a unit vector $\boldsymbol{n}$ orthogonal to the image of $H$ at $p$ with $\kappa_{g}=\boldsymbol{e} \cdot \boldsymbol{n}$. Conversely, if for some unit vector $\boldsymbol{n}$ at $f(p)$ one has $\left|\kappa_{g}\right|<\boldsymbol{e} \cdot \boldsymbol{n}$ then, at least with everything analytic, an isometric extension exists (Darboux [3, p. 274]). We shall show that for higher dimensional problems the analogous necessary curvature condition is also almost sufficient.

This paper was motivated by two questions. The first was to see how the geometric conditions necessary for the existence of an extension enter into the possibility of solving the initial value problem for a certain system of partial differential equations. This was not clear to the author in the proofs of either Cartan or Janet. The second was to see what modifications of the implicit function theorem of [7] were necessary in order to handle initial value theo-

Communicated by L. Nirenberg, December 7, 1972. This work was supported in part by NSF Grant GP-18961. 
rems. Indeed this paper, with just a little rewriting and generalization, could have been entitled "An implicit function theorem for initial value problems".

We need to introduce some notation and recall some definitions. The linear span of a set of vectors $\left\{v_{1}, \cdots, v_{k}\right\}$ is denoted by!1.s. $\left\{v_{1}, \cdots, v_{k}\right\}$. Let $\Phi: M^{n} \rightarrow E^{N}$ be an embedding of a manifold into Euclidean space. In a neighborhood of some point $p$ introduce local coordinates $x_{1}, \cdots, x_{n}$. The tangent space to the image of $M^{n}$ in $E^{N}$ at $p$ is denoted by $T_{p}(\Phi(M))$. Thus $T_{p}(\Phi(M))$ $=1$.s. $\left\{\Phi_{x_{1}}, \cdots, \Phi_{x_{n}}\right\}$. The osculating space at $p$ is defined by $\mathcal{O}_{p}(\Phi(M))=$ 1.s. $\left\{\Phi_{x 1}, \cdots, \Phi_{x_{n}}, \Phi_{x_{1} x_{1}}, \cdots, \Phi_{x_{r} x_{s}}, \cdots, \Phi_{x_{n} x_{n}}\right\}$. Both $T$ and $\mathcal{O}$ are independent of the choice of coordinates. The map $\Phi: M \rightarrow E^{N}$ is an immersion if dim $T_{p}(\Phi(M))=n$, and is nondegenerate if in addition $\operatorname{dim} \mathcal{O}_{p}(\Phi(M))=\frac{1}{2} n(n+3) ;$ in particular $N$ must be at least as large as this number. This is equivalent to the fact that the set of vectors occurring in the definition of $\mathcal{O}_{p}(\Phi(M))$ is linearly independent. If $\Phi$ is an immersion at $p$, then for some neighborhood of $p, \Phi$ is injective, i.e., is an embedding. Further, assuming $\Phi$ is an immersion, the usual metric on $E^{N}$ induces via $\Phi$ a Riemannian metric on $M$, to be denoted by $F(\Phi)$. Thus $F$ is a functional from immersions to metrics. We use the term general metric for a symmetric bilinear form which need be neither nondegenerate nor positive semi-definite. In local coordinates, $(F(\Phi))_{i j}=$ $\sum_{\nu=1}^{N}\left(\partial \Phi^{\nu} / \partial x_{i}\right)\left(\partial \Phi^{\nu} / \partial x_{j}\right)$. Given two maps $u$ and $v$ of $M$ into $E^{N}$ we may consider the Frechet derivative $d F(u) v$ of: $F$ at $u$ acting $v$. This derivative of $F$ at $u$ takes maps into general metrics: $(d F(u) v)_{i j}=\sum_{\nu=1}^{N}\left\{\left(\partial u^{\nu} / \partial x_{i}\right)\left(\partial v^{\nu} / \partial x_{j}\right)\right.$ $\left.+\left(\partial u^{\nu} / \partial x_{j}\right)\left(\partial v^{\nu} / \partial x_{i}\right)\right\}$. If $H$ is a submanifold of $M$, then everyone knows what the restriction of $v$ to $H$ is. We shall denote this by $\left.v\right|_{H}$. But for a metric $g$, by $\left.g\right|_{H}$ we do not mean the restriction of each component of $g$ to $H$ but rather the metric on $H$ induced by the inclusion of $H$ into $M$. If we choose local coordinates $x_{1}, \cdots, x_{n}$ such that $H=\left\{x \mid x_{n}=0\right\}$, then $\left(\left.g\right|_{H}\right)_{i j}=$ $g_{i j}\left(x_{1}, \cdots, x_{n-1}, 0\right), 1 \leq i \leq j \leq n-1$. Throughout this paper we use the indices $i$ and $j$ only in this range.

We shall often use the term isometry as an abbreviation for an isometric embedding $\Phi: M \rightarrow E^{N}$. This is not the usual usage. The problem of finding an isometric extension is the following: Given a manifold $U$ with Riemannian metric $g$ and a submanifold $H$ and a map $f: H \rightarrow E^{N}$ with $F(f)=\left.g\right|_{H}$, find a map $u: U \rightarrow E^{N}$ with $F(u)=g$ and $\left.u\right|_{H}=f$. This is an initial value problem for a nonlinear partial differential equation.

We solve this problem in the real analytic category by an application of the Cauchy-Kowalewski theorem. For the $C^{\infty}$ category we shall first try to solve the linearized initial value problem. Given a map $u$, can we find a solution $v$ to $d F(u) v=\Delta g$ such that $\left.v\right|_{H}=0$ ? We must require $\left.\Delta g\right|_{H}=0$. Here $\Delta g$ is used as symbol for a general metric. The notation anticipates our application. Note that we want to conclude that all the components of $v$ vanish on $H$, although we do not assume that $g_{i n}$ is zero on $H$. For this, Nash's method of inverting the linearized operator does not suffice, and so we seek to modify it. 
After doing this we attack the $C^{\infty}$ problem by first approximating it by a complex analytic one. This formalism involves the complexification of a manifold. If $M$ is a real analytic manifold of real dimension $n$, then there exists a complex analytic manifold $M^{C}$ of complex dimension $n$ such that $\mathrm{M}$ may be considered a real submanifold of $M^{C}$. For $U$ in $\boldsymbol{R}^{n}$ we take as its complexification any open set in $C^{n}$, which contains $U . A(r)$ denotes the set of functions (or maps, or metrics) analytic on $\left\{z \in C^{n} \mid\right.$ distance $\left.(\operatorname{Re} z, U)<r,|\operatorname{Im} z|<r\right\}$. For $v \in A(r)$, $|v|_{r, k}$ is the supremum of $v$ and its partial derivatives up to order $k$ over the above subset of $C^{n}$. Note the Cauchy estimate, $|v|_{r, k} \leq K r^{-k}|v|_{2 r, 0}$. Use $|v|_{r}$ in place of $|v|_{r, 0}$. We denote the ball in $C^{n}$ with radius $r$ and center at the origin by $B(r)$, and use $\tilde{B}(r)$ for the analogous ball in $\boldsymbol{R}^{n}$.

The extension of analytic isometric embeddings is studied in $\S 1$. In $\S 2$, the Cartan-Janet Theorem is proved. $\S 3$ provides the solution to the linearized problem; this is used in $\S 4$ to extend a restricted class of isometric embeddings. Then in the last section the results of $\S 1$ and $\S 4$ are combined to show that all appropriate isometric embeddings can be extended. These results are stated for codimension one submanifolds; similar results hold for arbitrary codimension. This section also includes a deformation result.

\section{Real analytic extensions}

Let $H$ be an $(n-1)$-dimensional submanifold of the $n$-dimensional Riemannian manifold $U$, and $f: H \rightarrow E^{N}$ be an isometry with respect to the metric $U$ induces on $H$. Fix a point $p \in H$, and identify the tangent spaces $T_{p} H$ and $T_{f(p)} f(H)$. Let $L: T_{p} H \times T_{p} H \rightarrow R^{1}$ and $\bar{L}: T_{p} H \times T_{p} H \rightarrow B$ be the second fundamental forms of $H$ in $U$ and $E^{N}$ respectively, where $B$ is the normal space to $f(H)$ at $f(p)$. If there exists an isometry $\tilde{f}: U \rightarrow E^{N}$ which extends $f$, then one has $\langle\nu, \bar{L}(X, Y)\rangle=L\langle X, Y\rangle$ for some unit vector $\nu$ in the tangent space to $E^{N}$ at $\tilde{f}(p)$ and for all $X$ and $Y$ in $T_{p} H$. Indeed $\nu=f_{*} \mu$, where $\mu \in T_{p} U, \mu \perp T_{p} H$, $\|\mu\|=1$, and $\mu$ has the appropriate orientation. In this way $\bar{L}$ necessarily dominates $L$. We want to study to what extent this domination is sufficient for the existence of such an extension.

Definition. For fixed $f$ and $p$ we say $\bar{L}>L$ if there exists some $\nu \in T_{f(p)} E^{N}$ with

1) $\|\nu\|<1$,

2) $\nu$ orthogonal to $T_{p} H$, and

3) $\langle\nu, \bar{L}(X, Y)\rangle=L(X, Y)$ for all $X$ and $Y$ in $T_{p} H$.

It is enough to require in 3) that $\left\langle\nu, \bar{L}\left(X_{i}, X_{j}\right)\right\rangle=L\left(X_{i}, X_{j}\right)$ for some basis $X_{1}, \cdots, X_{n-1}$ of $T_{p} H$. Take $N \geq \frac{1}{2} n(n+1)$. If $\bar{L}>L$ for $f$ and $p$, and $f$ is nondegenerate, then the same is true for $f^{\prime}$ and $p^{\prime}$ with $f^{\prime}$ near $f$ in $C^{2}$ and $p^{\prime}$ close enough to $p$. Also there is a unique vector satisfying 2) and 3) with smallest norm. This vector varies smoothly as a function of $p$. Both of these follow since for $f$ nondegenerate $\mathcal{O}_{p}(f(H))$ is maximal (i.e., of dimension 
$\left.\frac{1}{2} n(n+1)-1\right)$. Further by adding to $\nu$ a vector perpendicular to $\mathcal{O}_{p}(f(H))$ we can obtain a smoothly varying unit vector satisfying 2 ) and 3 ).

We note the following for use in $\S 2$. Let $H$ be geodesic in the sense of being the image under $\exp _{p}: T_{p} U \rightarrow U$ of an $(n-1)$-dimensional linear subspace. Then in the natural coordinates all the Christoffel symbols of the connection vanish at $p$, and at this point $L=0$. Thus for any isometry $f: H \rightarrow E^{N}$ one has $\bar{L}>L$.

Recall that the existence of an extension only implied one could find a solution to 2) and 3) with $\|\nu\|=1$. Substituting $\|\nu\| \leq 1$ for the strict inequality we could have defined $\bar{L} \geq L$. However $\bar{L} \geq L$ is clearly not sufficient for the existence of an extension as the following example shows.

We compare the metric $d s_{\Phi}^{2}=d r^{2}+(\Phi(r) r)^{2} d \theta^{2}$ on $R^{2}$ with the standard metric $d s^{2}$ on $E^{2}$ corresponding to $\Phi \equiv 1$. Let $\Phi(1)=1, \Phi^{\prime}(1)=0, \Phi^{\prime \prime}(1)<0$. Consider two points belonging to the unit circle and close together. Clearly the distance between these two points in the first metric in strictly less than their distance in the standard metric. The inclusion $S^{1} \rightarrow E^{2}$ of the unit circle is isometric, since $d s_{\Phi}^{2}=d s^{2}$ on $S^{1}$, and, by what we have just said, cannot be extended in a neighborhood of any point to an isometry.

The same is clearly true for this map thought of as a map of $S^{1} \rightarrow E^{k}$. In particular, set $f(\theta)=(\cos \theta, \sin \theta, 0)$, and let $x_{1}=r, x_{2}=\theta$. Thus $\Gamma_{22}^{1}=-1$. To find $\nu$ we need $\nu \cdot f_{x_{2}}=0$ and $\nu \cdot f_{x_{2} x_{2}}=-1$. We can find a solution with $\|\nu\|=1$ (but none with $\|\nu\|<1$ ). Thus $\bar{L} \geq L$ is not sufficient for the existence of an extension.

We investigate what $\bar{L}>L$ means in terms of local coordinates. Introduce coordinates $x_{1}, \cdots, x_{n-1}, y=x_{n}$ on $U$ such that $H=\{(y, x) \mid y=0\}$ and the metric satisfies $g_{n i}=0, g_{n n}=1$. In this paper always $1 \leq i \leq j \leq n-1$. The vector fields $Y, X_{1}, \cdots, X_{n-1}$ denote the tangent vectors to the appropriate curves. One computes that the Christoffel symbol $\Gamma_{i j}^{n}$ is equal to $-\frac{1}{2} g_{i j, n}$. Thus $L_{i j}=\left\langle Y, X_{i} \vdash X_{j}\right\rangle=-\frac{1}{2} g_{i j, n}$. From $\left.\bar{L}\right\rangle L$ we know there exists a vector $\nu\left(p^{\prime}\right) \in T_{p^{\prime}} E^{N}$ (which we henceforth identify with $E^{N}$ ) which is of unit length, satisfies 2) and 3) and varies analytically as a function of $p^{\prime}$. In terms of the map $f: H \rightarrow E^{N}$ we have $\nu \cdot f_{x_{i} x_{j}}=-\frac{1}{2} g_{i j, n}, \nu \cdot f_{x_{i}}=0$ and $\nu \cdot \nu=1$. We shall now see that for real analytic Riemannian manifolds the existence of a solution to this algebraic system implies the existence of an isometric map $\tilde{f}: U \rightarrow E^{N}$ which extends $f$.

Theorem 1.1. Let $U$ be a real analytic Riemannian manifold of dimension $n, H$ an analytic submanifold of codimension one with the induced metric, and $p$ a point of $H$. Assume there is a map $f: H \rightarrow E^{N}, N=\frac{1}{2} n(n+1)$, which in a neighborhood of $p$ is analytic and isometric. Finally assume that at $p, f$ is nondegenerate and $\bar{L}>L$. Then one can find an open neighborhood $\tilde{U}$ of $p$ in $U$ and a real analytic map $\tilde{f}: \tilde{U} \rightarrow E^{N}$ such that $\tilde{f}$ is an isometric embedding and $\tilde{f}$ agrees with $f$ on $H \cap \tilde{U}$. Further the augmented osculating space of $\boldsymbol{H}$ 
with respect to $\tilde{f}$ is maximal. If $N \geq \frac{1}{2} n(n+3)$, then $\tilde{f}$ can be chosen to be nondegenerate on $\tilde{U}$.

This augmented osculating space is the linear span of $\mathcal{O}_{p}(f(H))$ and $T_{p}(\tilde{f}(U))$. Its maximality reflects our choice below of an initial vector $f_{y}(p)$ which does not lie in $\mathcal{O}_{p}(f(H))$. The fact that $\tilde{f}$ is nondegenerate if the dimension $N$ is high enough is crucial for our proofs of both the Cartan-Janet theorem and the $C^{\infty}$ extension result.

Proof. In the local coordinates introduced above, the isometric embedding equations become

$$
f_{x_{i}} \cdot f_{x_{j}}=g_{i j}, \quad f_{x_{i}} \cdot f_{y}=0, \quad f_{y} \cdot f_{y}=1 .
$$

We consider $f\left(0, x_{1}, \cdots, x_{n-1}\right)$ as given and seek a solution $f\left(y, x_{1}, \cdots, x_{n-1}\right)$ $=f(0, x)+f_{1}(x) y+f_{2}(x) y^{2}+\cdots$. Equivalently we reduce (1) to a system to which we may apply the Cauchy-Kowalewski theorem. It is easy to see that a solution of (1) must also satisfy

$$
\begin{gathered}
f_{y} \cdot f_{x_{i} x_{j}}=-\frac{1}{2}\left(g_{i j}\right)_{y}, \quad f_{y} \cdot f_{x_{i}}=0, \quad f_{y} \cdot f_{y}=1, \\
f_{y y} \cdot f_{x_{i}}=0, \quad f_{y y} \cdot f_{y}=0, \quad f_{y y} \cdot f_{x_{i} x_{j}}=\frac{1}{2}\left(g_{i j}\right)_{y y}+f_{y x_{i}} \cdot f_{y x_{j}} .
\end{gathered}
$$

We have already seen that the condition on the second fundamental forms implies that $f_{y}(0, x)=\nu(x)$ satisfies (2) on $H$. Further $\nu(x)$ is linearly independent of $\mathcal{O}(H)$, i.e., the matrix whose rows are $\nu, f_{x_{i}}, \cdots, f_{x_{n-1}}, f_{x_{1} x_{1}}, \cdots, f_{x_{i} x_{j}}$, $\cdots, f_{x_{n-1} x_{n-1}}$ is invertible. Thus we can apply the Cauchy-Kowalewski theorem to system (3) with $f(0, x)$ the given map of $H$ into $E^{N}$ and $f_{y}(0, x)$ equal to $\nu(x)$. This means applying the Cauchy-Kowalewski theorem to a possibly underdetermined system. We will comment on this later.

It is now straightforward to verify that the analytic function $f(x, y)$ so obtained also satisfies (1). We do the longest of the calculations: Assume we have already shown $f_{x_{i}} \cdot f_{y}=0$ and $f_{y} \cdot f_{y}=1$ wherever $f(y, x)$ is defined and for all $i=1, \cdots, n-1$. For some $i$ and $j$, set $u=x_{i}, v=x_{j}$. On $H$ we have $f_{u} \cdot f_{v}$ $=g_{i j}$, and want to obtain $f_{u} \cdot f_{v}=g_{i j}$ everywhere. We know that on $H, f$ satisfies the initial condition $f_{y} \cdot f_{u v}=-\frac{1}{2}\left(g_{i j}\right)_{y}$. Differentiating $f_{u} \cdot f_{y}=0=$ $f_{v} \cdot f_{y}$ gives that $\left(f_{u} \cdot f_{v}\right)_{y}=-2 f_{u v} \cdot f_{y}=\left(g_{i j}\right)_{y}$ on $H$. If we can now show that $\left(f_{u} \cdot f_{v}\right)_{y y}=\left(g_{i j}\right)_{y y}$ everywhere, then clearly $f_{u} \cdot f_{v}=g_{i j}$ everywhere. From $f_{u} \cdot f_{y}$ $=f_{v} \cdot f_{y}=0$ and $f_{y} \cdot f_{y}=1$ we derive $f_{u y y} f_{v}=-f_{u v y} f_{y}-f_{u y} f_{v y}-f_{u v} f_{y y}$ and $f_{u v y} f_{y}=-f_{u y} f_{v y}$. Thus using (2) we obtain $\left(f_{u} \cdot f_{v}\right)_{y y}=\left(g_{i j}\right)_{y y}$, which implies that a solution to system (3) satisfying the above initial conditions is also a solution to system (1), i.e., provides an extension of the isometric embedding $H \rightarrow E^{N}$. Clearly for this extension we have a maximal augmented osculating space of $H$ in $E^{N}$. We want to show that for $N \geq \frac{1}{2} n(n+3)$ some nondegenerate extension can be found.

Let $B\left(\lambda_{1}, \cdots, \lambda_{k}\right)$ be an $r \times s$ matrix, $r<s$, depending analytically on para- 
meters $\lambda_{i}$ and of maximal rank, and $C\left(\lambda_{1}, \cdots, \lambda_{k}\right)$ be some right inverse also varying analytically. Now consider an equation $B\left(f_{x_{I}}, f_{x_{I} x_{J}}\right) f_{y y}=g$. Then any solution to $f_{y y}=C\left(f_{x_{I}}, f_{x_{I} x_{J}}\right) g$ also satisfies $B f_{y y}=g$; this is how the CauchyKowalewski theorem is applied to underdetermined systems. We want to modify this so as to obtain nondegenerate isometries.

Given a set $S=\left\{v_{1}, \cdots, v_{k}\right\}$ of vectors in $E^{N}, N>k$, let $\mu\left(v_{1}, \cdots, v_{k}\right)$ be some nonzero vector orthogonal to $S$. If each $v_{i}$ varies analytically, and $S$ is a linearly independent set, then $\mu$ can also be chosen analytic. If $B$ is now a matrix whose rows are given by the vectors $f_{x_{i}}, f_{y}, f_{x_{i} x_{j}}$, and $C$ is again some right inverse, while $\mu=\mu\left(f_{x_{i}}, f_{y}, f_{x_{i} x_{j}}\right)$, then $f_{y y}=C g+\mu$ also satisfies $B f_{y y}$ $=g$. Using this observation we can insure nondegeneracy. First take $C$ to be that right inverse whose range is the linear span of the rows of $B$. Next we use the following lemma to help find an appropriate $\mu$.

Lemma. If $\bar{L}>L$ at the origin, and $N \geq \frac{1}{2} n(n+3)$, then there exists a vector $\nu(x)$ in $E^{N}$ analytic near the origin, for which $f_{y}(x, 0)=\nu(x)$ satisfies (2) and $\left\{\nu, \nu_{x_{i}}, f_{x_{i}}, f_{x_{i} x_{j}}\right\}$ is linearly independent at the origin.

For the proof find some $\nu_{1}$ such that (2) is satisfied, except $\left|\nu_{1}\right|<1$. Seek $\nu(x)=\nu_{1}(x)+\alpha_{0}(x)+\beta(x)\left(x_{1} \alpha_{1}(x)+\cdots+x_{n-1} \alpha_{n-1}(x)\right)$ where each $\alpha_{p}(x)$ is either zero or orthogonal to $\mathcal{O}(H)$. Choose $\beta(x)$ so $|\nu(x)|=1$. It is not too difficult to see that it is possible to pick the $\alpha$ 's so that $\left\{\nu, \nu_{x_{i}}, f_{x_{i}}, f_{x_{p} x_{q}}\right\}$ is linearly independent at the origin.

Now consider $\mu\left(v_{1}, \cdots, v_{k}\right)$ as above. In particular we will look at $\mu\left(f_{x_{i}}, f_{x_{i} x_{j}}, f_{y}, f_{y x_{i}}\right)$ where, because of the initial data $f_{y}=\nu$ and the above lemma, we can take $\mu$ to be an analytic function of its arguments. The solution to $f_{y y}=C\left(f_{x_{i}}, f_{y}, f_{x_{i} x_{j}}\right) g+\mu\left(f_{x_{i}}, f_{y}, f_{x_{i} x_{j}}, f_{y x_{i}}\right)$, with the initial data $f(x, 0)=$ the given embedding of $H$ and $f_{y}(x, 0)=\nu$, also satisfies $B f_{y y}=g$ and so, as we have seen, also satisfies (1), i.e., gives an isometric embedding. Note $f_{y y}$ is linearly independent of $\left\{f_{x_{i}}, f_{y}, f_{x_{i} x_{j}} f_{y x_{k}}\right\}$, so that $\operatorname{dim} \mathcal{O}(f(U))=\frac{1}{2} n(n+3)$ and $f$ is nondegenerate. This concludes the proof of Theorem 1.1.

This theorem is a local result. We can also ask when the isometry $f: H \rightarrow E^{N}$ can be extended to a neighborhood of all of $H$. So let $f$ be nondegenerate, and suppose $\bar{L}>L$ everywhere on $H$. Note that once the initial condition $f_{y}(x, 0)$ is given, the solution to system (3) given by the Cauchy-Kowalewski theorem is unique and so is independent of the choice of coordinates $x_{1}, \cdots, x_{n-1}$. Let $B$ be the line bundle on $H$ determined by the direction orthogonal to $\mathcal{O}(f(H))$, and $D$ be the normal line bundle of $H$ in $U$. If $B$ and $D$ are isomorphic, then the initial data can be globally prescribed. Hence, if $H$ is also closed in $U$, then the isometric embedding $f$ can be extended to an isometric embedding of an open neighborhood of $H$. Of course, $B$ and $D$ isomorphic is also necessary for the existence of an extension for which $H$ has everywhere an augmented osculating space of maximum rank. A more complicated condition arises when one requires that the extension be nondegenerate.

The main result of this paper is to show that even nonanalytic isometric 
embeddings can be extended, provided we replace $N \geq \frac{1}{2} n(n+1)$ by $N \geq$ $\frac{1}{2} n(n+1)+n$. Before doing this we detour to show how one may now prove that any real analytic $n$-dimensional manifold has a local analytic isometric embedding into $E^{N}$. We also discuss previous work on this real analytic problem.

\section{The Cartan-Janet theorem and some generalizations}

In this section we prove several well known results. Let us start with the Cartan-Janet Theorem.

Theorem 2.1. Let $U$ be an $n$-dimensional real analytic Riemannian manifold, and $p$ a point of $U$. Then there exist an open set $\tilde{U}$ containing $p$ and a real analytic map of $\tilde{U}$ into $E^{N}, N=\frac{1}{2} n(n+1)$, which is an isometric embedding.

Let $p \in H^{1} \subset H^{2} \subset \cdots \subset H^{n-1} \subset U$ be a sequence of analytic submanifolds, $\operatorname{dim} H^{k}=k$, We seek analytic nondegenerate isometries of each $H^{k}$ (or rather an open subset of $H^{k}$ containing $p$ ) into $E^{N}, N=\frac{1}{2} n(n+1)$. For $H^{1}$ this is simple since the introduction of arc length is an analytic change of variables. If $H^{k-1}$ is so embedded, and our curvature condition holds, then one may apply Theorem 1.1 and obtain a similar embedding for $H^{k}$. At the last step we obtain an embedding of $U$ which proves Theorem 2.1, or we can replace $N$ by $\frac{1}{2} n(n+3)$ and obtain a variant of this theorem: Every such $U$ has an analytic nondegenerate isometric embedding into $E^{N}, N=\frac{1}{2} n(n+3)$.

Before questioning whether the sequence of manifolds $H^{k}$ and their nondegenerate embeddings can be found such that the curvature restriction is valid, recall that this restriction is always fulfilled if the manifolds are geodesic at $p$. This proves Theorem 2.1.

Let $M$ and $\bar{M}$ be Riemannian manifolds, $\operatorname{dim} M=n, \operatorname{dim} \bar{M}=\frac{1}{2} n(n+1)$. Let $H$ be a submanifold of codimension one in $M$, and $h: H \rightarrow \bar{M}$ an isometry. We wish to extend $h$ to an isometry $f: M \rightarrow \bar{M}$. Again we assume everything is real analytic and seek only local results. For local coordinates on $M$ we wish to solve

$$
\left\langle f_{*}\left(\frac{\partial}{\partial x_{i}}\right), f_{*}\left(\frac{\partial}{\partial x_{j}}\right)\right\rangle=\left\langle\frac{\partial}{\partial x_{i}}, \frac{\partial}{\partial x_{j}}\right\rangle,
$$

where the first inner product is in $\bar{M}$, and the second in $M$. Let $H=\left\{x \mid x_{n}=0\right\}$, and take $\left\langle\partial / \partial x_{n}, \partial / \partial x_{i}\right\rangle=0, i \neq n$. Also set $X_{i}=f_{*}\left(\partial / \partial x_{i}\right)$. A necessary condition for an isometric extension to exist is that there exists $W \in T \bar{M}$ with $\langle W, W\rangle=1,\left\langle W, X_{i}\right\rangle=0$, and $\left\langle W, \bar{D}_{X_{i}} X_{j}\right\rangle=\Gamma_{i j}^{n}$ along $h(H) \subset \bar{M}$. We still use $1 \leq i \leq j<n$. Here $\bar{\nabla}$ is the connection in $\bar{M}$ (subsequently also denoted by $\vdash$ ), and $\Gamma_{i j}^{n}$ the Christoffel symbol in $M$. It is easy to verify that any solution to

$$
\left\langle\bar{\nabla}_{X_{n}} X_{n}, X_{i}\right\rangle=0, \quad\left\langle\bar{\nabla}_{X_{n}} X_{n}, X_{n}\right\rangle=0,
$$




$$
\begin{aligned}
\left\langle\bar{\nabla}_{X_{n}} X_{n}, \bar{\nabla}_{X_{i}} X_{j}\right\rangle= & -\frac{1}{2} \frac{\partial^{2}}{\partial x_{n}^{2}}\left\langle\frac{\partial}{\partial x_{i}}, \frac{\partial}{\partial x_{j}}\right\rangle+\left\langle\dot{\nabla}_{X_{n}} X_{i}, \bar{\nabla}_{X_{n}} X_{j}\right\rangle \\
& +\left\langle R\left(X_{n}, X_{i}\right) X_{j}, X_{n}\right\rangle
\end{aligned}
$$

subject to the initial conditions on $H$

$$
\left\langle X_{n}, \bar{\nabla}_{X_{i}} X_{j}\right\rangle=\Gamma_{i j}^{n},\left\langle X_{n}, X_{i}\right\rangle=0,\left\langle X_{n}, X_{n}\right\rangle=1
$$

also gives a solution to (1). Here $R$ is the curvature form $R(A, B) C=-A \vdash$ $(B \vdash C)+B \vdash(A \vdash C)+[A, B] \vdash C$.

Using local coordinates and recalling that $R$ is a tensor and hence does not depend on the derivatives of $f$, we see that the Cauchy-Kowalewski theorem can be applied provided $\left\{X_{i}, X_{n}, \bar{\nabla}_{X_{i}} X_{j}\right\}$ are linearly independent on the initial surface $H$. So we say $f$ is nondegenerate at $p \in H$ if the set $\left\{X_{i}, \vec{\nabla}_{X_{i}} X_{j}\right\} \subset$ $T_{f(p)} \bar{M}$ is linearly independent, and we say $\bar{L}>L$ if there exists $W \in T \bar{M}_{f(p)}$ with $\langle W, W\rangle\left\langle 1,\left\langle W, X_{i}\right\rangle=0\right.$, and $\left\langle W, \bar{\nabla}_{X_{i}} X_{j}\right\rangle=L\left(X_{i}, X_{j}\right), L$ being the second fundamental form of $H$ in $M$.

Theorem. If $f$ is nondegenerate, and $\bar{L}>L$ at $p \in H$, then the isometry $h: H \rightarrow \bar{M}$ can be extended to an analytic isometry in a neighborhood in $M$ of $p$.

As before, we note that if $H$ is a geodesic submanifold of $M$ at $p$, then any isometry $h: H \rightarrow \bar{M}$ can be extended, provided that $h$ is nondegenerate. Also note that if $\bar{M}$ is a submanifold of some $\tilde{M}$, geodesic at some point $q$, then for any $h: M \rightarrow \bar{M}$ with $h(p)=q$ the osculating space of $h(H)$ at $q$ as a linear subspace of $T_{q} \tilde{M}$ is actually contained in $T_{q} \bar{M}$. With these two observations one can prove the following generalization of the Cartan-Janet Theorem.

Theorem 2.2. Let $M_{1}$ and $M_{2}$ be arbitrary analytic Riemannian manifolds of dimensions $n$ and $\frac{1}{2} n(n+1)$ respectively. Then about each point in $M_{1}$ there exists an open set which can be mapped isometrically into $M_{2}$ by a real analytic map.

We now consider metrics which are indefinite but nondegenerate. This means that if we diagonalize the metric at a point, we obtain positive and negative eigenvalues but no zero ones. Note however that we can obtain nonzero tangent vectors of zero norm. Such directions are said to be isotropic. A metric with $n_{1}$ positive and $n_{2}$ negative eigenvalues is said to be of type $\left(n_{1}, n_{2}\right)$, and the underlying manifold is denoted by $M^{n_{1}, n_{2}}$ or $M^{n}, n=n_{1}+n_{2}$, when type is not important. Let $H^{n-1}\left(=H^{n_{1}, n_{2}}\right)$ be a submanifold of $U^{n}, p$ a point of $H$, and $f: H^{n-1} \rightarrow E^{N}, N=\frac{1}{2} n(n+1)$, a nondegenerate isometry. Again everything is real analytic, and we seek a real analytic isometric extension of $f$ in a neighborhood of $p \in U$.

Note that for any set $S$ of $k-1$ linearly independent vectors in an indefinite space $E^{k}$ there is a vector orthogonal to $S$ (although it may also be an element of $S$ ). Further this vector is unique up to scalar multiplication. So upon fixing 
a direction, let $t_{U}$ denote the vector normal to $H$ in $U$, and let $t_{E}$ denote the vector normal to $\mathcal{O}(H)$ in $E^{N}$. Let $\sigma_{U}= \pm 1$ if there exists $\lambda$ such that $\left\|\lambda t_{U}\right\|=$ \pm 1 , and $\sigma_{U}=0$ otherwise, and the same for $\sigma_{E}$. We shall always assume $\sigma_{U} \neq 0$, i.e., the direction orthogonal to $H$ is nonisotropic. This allows us to introduce local coordinates in the same way as before. Let $v \in \mathcal{O}(f(H))$ be that unique element with $f_{x_{i}} \cdot v=0$, and $f_{x_{i} x_{j}} \cdot v=L\left(X_{i}, X_{j}\right)$.

Theorem. The nondegenerate map $f: H^{n} \rightarrow E^{N}$ has an isometric extesion under any of the following conditions

(i) $\|v\|<-1$ and $\sigma_{E}>0$,

(ii) $\|v\|>1$ and $\sigma_{E}<0$,

(iii) $-1<\|v\|<1$ and $\sigma_{E} \sigma_{L I}>0$.

The proof is clear. The fact that the metric is indefinite play no role in solving system (3) but only in satisfying the initial conditions (2). The inner product $u \cdot v$ now has the form $\sum_{\alpha=1}^{k} u^{\alpha} v^{\alpha}-\sum_{\alpha=k+1}^{N} u^{\alpha} v^{\alpha}$ where $E^{N}=E^{k, N-k}$.

Corollary. Suppose that $H^{n, m} \rightarrow E^{r, s}$ is a nondegenerate isometry $(r+s=$ $\left.\frac{1}{2}(n+m)(n+m+1)-1\right)$, and that $H^{n, m}$ is geodesic in $U^{n, m+1}\left(\right.$ or $\left.U^{n+1, m}\right)$. Then one can extend to an isometry $U^{n, m+1} \rightarrow E^{r, s+1}\left(\right.$ or $\left.U^{n+1, m} \rightarrow E^{r+1, s}\right)$ with maximum osculating space if and only if $\sigma_{U}<0\left(\right.$ or $\left.\sigma_{U}>0\right)$.

Call $E^{N}$ compatible with $H^{n}$ if $E^{N}$ is of type $\left(N_{1}, N_{2}\right)$, and $H^{n}$ is of type $\left(n_{1}, n_{2}\right)$ with $N_{1} \geq n_{1}, N_{2} \geq n_{2}$.

Lemma. $H^{n}$ has an isometric nondegenerate embedding into $E^{N}, N \geq$ $\frac{1}{2} n(n+3)$, if and only if $E^{N}$ is compatible with $H^{n}$.

So if we apply the above results to some $H^{n-1}$ which is geodesic in $U^{n}$ and whose orthogonal direction is nonisotropic we obtain the following result.

Theorem 2.3. $U^{n}$ can be locally embedded in $E^{N}, N=\frac{1}{2} n(n+1)$, by a real analytic isometry provided only $E^{N}$ is compatible with $U^{n}$.

One can naturally also replace the Euclidean indefinite metric by any other nondegenerate one.

Theorem 2.1 was first proved for two-dimensional manifolds in Euclidean three-space by Weingarten [12]. Janet [9] in 1926 sketched a proof of the general case; this was incomplete because he could not guarantee the existence of the appropriate initial conditions. Burstein [1] in 1931 completed Janet's proof, and stated that the generalization to Theorem 2.2 presents no difficulty. He showed that $f: H^{n-1} \rightarrow E^{N}, N=\frac{1}{2} n(n+1)$, could be extended whenever $f$ was nondegenerate and $H^{n-1}$ geodesic. The use of such geodesic hypersurfaces obscured the curvature condition and its relation to the initial values. In the meantime Cartan [2] published a proof of Theorem 2.2 using his theory of exterior differential forms. In 1956 Leichtweiss [10] gave a new proof of Theorem 2.2 in the spirit of Janet's and Burstein's work. Finally, Theorem 2.3 is due to Friedman [5], using another variation of Janet's idea. This result for indefinite metrics was known to Eisenhart [4, p.188].

We have also used this basic idea of reducing the isometric embedding equations to a system of Cauchy-Kowalewski type. The material in sections one and 
two differs from that of previous authors in that it focuses on the necessary geometric conditions for extending a given embedding of some (not necessarily geodesic) submanifold. This viewpoint seems to make the proofs simpler and more natural.

One can also consider nonanalytic Riemannian manifolds.

Theorem. Let $U^{n}$ be a Riemannian manifold of class $C^{\alpha}, \alpha>2$. Then each point of $U^{n}$ has an open neighborhood which admits an isometric embedding of class $C^{\alpha}$ into $E^{N}, N=\frac{1}{2} n(n+1)+n$.

This follows from the arguments used by Nash [11] in his work on global isometric embeddings. The $C^{\infty}$ case was proved by Greene [6], and the general result can be easily proved using [7].

However these techniques are not immediately applicable to extension problems, i.e., to initial value problems. It is the aim of the present paper to show what modifications are necessary, or rather sufficient. Of course once we solve the extension problem for nonanalytic metrics, we can prove corresponding version of the above theorem in the same way as we proved the Cartan-Janet theorem, but we do not in this way get that the embedding is also of class $C^{\alpha}$.

\section{The linearized equations}

Our goal is to generalize Theorem 1.1 to nonanalytic metrics and embeddings. The first step is to study the linearization $d F(u) v=\Delta g$ defined in the introduction. For $N>\frac{1}{2} n(n+1)$ the equations are underdetermined, and so we may assume that certain additional conditions on $v$ are also valid. If $N \geq$ $\frac{1}{2} n(n+1)+n$, and the map $u: U \rightarrow E^{N}$ is nondegenerate, then, as Nash showed [11, pp. 31-34], there exists one and only one solution $v$ also satisfying $v \in$ $\mathcal{O}(U) \cap(T(U))^{\perp}$. We cannot use this construction since, for this inverse to the linear operator, $d F(u) v=\Delta g$ and $\left.\Delta g\right|_{H}=0$ do not imply $v=0$ on $H$. Thus $v$ does not solve the linearized initial value problem. So we modify Nash's solution. We pay for this modification by not retaining as good a bound on $v$.

Let $u: U \rightarrow E^{N}$ be a given map. Again we use the indices $1 \leq i \leq j<n$, and introduce coordinates $x_{1}, \cdots, x_{n}$ such that $g_{n n}=1$ and $g_{n i}=0$, i.e., $u_{x_{n}} \cdot u_{x_{n}}=1$ and $u_{x_{i}} \cdot u_{x_{n}}=0$. We later also use the indices $1 \leq I \leq J \leq n$. Assume the submanifold $H$ is given by $\left\{x \mid x_{n}=0\right\}$. Set $\nu=\partial / \partial x_{n}$, denote 1.s. $\{\tilde{L}(\nu, x) \mid x \in T U\}$ by $S$, where $\tilde{L}(x, y)$ is the second fundamental form of $U$ in $E^{N}$, and set $\mathcal{O}=T(U) \oplus\left(\mathcal{O}(U) \cap(S \oplus T(U))^{\perp}\right)$. If we make some canonical choice of the coordinate $x_{n}$, then this space $\mathcal{O}$ is independent of the choice of (the other) coordinates. So let the coordinate curve of $x_{n}$ always be the unique geodesic orthogonal to $H$.

Let $h: H \rightarrow T U$ be any section, and $\Delta g$ any general metric. The notation $\Delta g$ suggests our future application.

Lemma. Assume $u$ is nondegenerate, and $N \geq \frac{1}{2} n(n+3)$. If $\left.\Delta g\right|_{H}=$ $\left.d F(u) h\right|_{H}$, then the system of differential equations $d F(u) v=\Delta g$, together with 
the conditions $v=h$ on $H$ and $v \in \mathcal{O}$ everywhere, have a unique solution.

Proof. Let $v \in \mathcal{O}$, and consider the unique decomposition $v=a+z, a \in$ $T(U), z \in \mathcal{O} \cap T(U)^{\perp}$. Set $a=a^{I} u_{x_{I}}$, (summation convention with $i$ or $j$ summed from 1 to $n-1$ and $I$ or $J$ from 1 to $n$; but if an index appears on both sides of an equation, then no summation is intended).

The eqations $d F(u) v=\Delta g$ become

$$
\begin{gathered}
2 a_{x_{n}}^{n}=\Delta g_{n n}, \quad g_{i j} a_{x_{n}}^{j}+a_{x_{i}}^{n}=\Delta g_{n i}, \\
-2 u_{x_{i} x_{j}} \cdot z=\Delta g_{i j}-(d F(u) a)_{i j} .
\end{gathered}
$$

We have used the obvious equalities:

$$
\begin{gathered}
u_{x_{n}} \cdot u_{x_{I} x_{n}}=0, \quad u_{x_{n}} \cdot z_{x_{I}}=0=u_{x_{I}} \cdot z_{x_{n}} \\
u_{x_{n}} \cdot u_{x_{I} x_{J}}+u_{x_{I}} \cdot u_{x_{J} x_{n}}=0, \quad u_{x_{I}} \cdot z_{x_{J}}=-u_{x_{I} x_{J}} \cdot z .
\end{gathered}
$$

There is a unique solution to the first $n$ equations which agrees with $h$ on $H$. This solution satisfies the estimates $\left|a^{n}\right|<C\{|\Delta g|+|h|\}$ and $\left|a^{i}\right|<$ $C\left\{|\Delta g|_{1}+|h|_{1}\right\}$. The norm without a subscript is the maximum norm taken over some domain, and the other is the maximum of this norm, for the function and all of its first derivatives, taken over the same domain. We wish to solve the remaining $\frac{1}{2} n(n-1)$ equations for $z$ and to have $z=0$ on $H$.

Lemma. If $u$ is nondegenerate, then at each point of $U$ the linear algebraic system $u_{x_{i} x_{j}} \cdot z=G_{i j}$ has a unique solution for any right hand side. This solution varies analytically if $u$ and $G$ are analytic.

In our application, $-2 G_{i j}=\Delta g_{i j}-(d F(u) a)_{i j}$ where, on $H, a=h$ and $d F(u) h=\Delta g_{i j}$. So indeed $z=0$ on $H$.

To prove the lemma we note that the orthogonal projection of $\mathcal{O} \cap T(U)^{\perp}$ into 1.s. $\left\{u_{x_{i} x_{j}}\right\}$ is always injective, while $\operatorname{dim} \mathcal{O}(U)=\frac{1}{2} n(n+3)$ implies dim $\mathcal{O} \cap T(U)^{\perp}=\frac{1}{2} n(n-1)$, so that this map is surjective and 1.s. $\left\{u_{x_{i} x_{j}}\right\}$ is of maximal dimension. Thus one has a unique solution of $u_{x_{i} x_{j}} \cdot z=G_{i j}$ under the side condition $z \in \mathcal{O} \cap T(U)^{\perp}=\mathcal{O}(U) \cap S^{\perp} \cap T(U)^{\perp}$.

We now introduce the complexification of $U$ and the associated norms as outlined in the introduction. It is easy to see that our previous results carry over.

Theorem. Let $u^{0}: U^{C} \rightarrow C^{N}$ be analytic and nondegenerate in some complex ball $B\left(r_{0}\right)$. Given $\Delta g$ also analytic on $B\left(r_{0}\right)$ with $\Delta g=0$ on $H$, one can find $v^{0} \in A\left(r_{0}\right)$ satisfying $d F\left(u^{0}\right) v^{0}=\Delta g, v^{0}=0$ on $H$. Further there are local coordinates depending on $u^{0}$ and a constant $K$ also depending on $u^{0}$ such that for every $r$ with $0<r \leq r_{0}$ one has $\left|v^{0}\right|_{r}<K|\Delta g|_{r, 2}$.

The estimate on $\left|v^{0}\right|_{r}=|a+z|_{r}$ comes from the complex form of our previous estimate for $a$ and the observation that $z=M\left(\Delta g-d F\left(u^{0}\right) a\right)$ where $M$ is a matrix depending only on $\left\{u_{x_{I} x_{J}}\right\}$.

Let $u \in A\left(r_{0}\right)$. For $\left|u-u^{0}\right|_{r_{0}, 2}$ small, $u$ is also nondegenerate, and so we can 
find a corresponding $v$. We can even assume that the same $K$ suffices. So we get $|v|_{r} \leq K|\Delta g|_{r, 2}$ with respect to coordinates whose choice depends on $u$. Does this inequality also hold for the original coordinates depending on $u^{0}$ ?

Lemma. There exists some $\varepsilon>0$ such that $\left|u^{0}-u\right|_{r .4}<\varepsilon$ implies $|v|_{r} \leq$ $K|\Delta g|_{r, 2}$ even in the original coordinates.

To prove this one just has to study how the special coordinates we have introduced depend on the choice of metric. This is a straightforward application of the fundamental theorem of ordinary differential equations on the local existence of solutions and their dependence on parameters.

So we start with $u^{0}$ and fix a choice of coordinates. We have proved the following.

Theorem 3.1. Let $u^{0}: U \rightarrow E^{N}$ be a nondegenerate analytic embedding and $H$ an analytic submanifold of $U$. Then there exist positive numbers $\varepsilon, r_{0}, K$ such that if $u \in A(r)$ and $\Delta g \in A(r)$ for some $r<r_{0}$ with $\left|u-u^{0}\right|_{r, 4}<\varepsilon$ and $\left.\Delta g\right|_{H}$ $=0$, then there exists $v \in A(r)$ satisfying $d F(u) v=\Delta g, v=0$ on $H$, and $|v|_{r}<K|\Delta g|_{r, 2}$.

We use the following notation. Let $d F^{-1}(u)$ denote the operator defined by $d F^{-1}(u) \Delta g=v$ for that $v$ given by Theorem 3.1. This is a right inverse for $d F(u)$ and $\left|d F^{-1}(u) \Delta g\right|_{r}<K|\Delta g|_{r, 2}$. The operator $d F^{-1}(u)$ is itself Frechet differentiable; we use this at the end of $\S 5$ in connection with deformation results.

\section{Extension of certain isometric embeddings}

In this section we show that even nonanalytic isometric embeddings $f: H \rightarrow$ $E^{N}$ can be extended as long as they are close to analytic nondegenerate embeddings of $U$. Without loss of generality take in place of $U$ the ball $\tilde{B}(R)$ in $\boldsymbol{R}^{n}$ with radius $R$ and center at the origin. Assume $u: U \rightarrow E^{N}, N \geq \frac{1}{2} n(n+3)$, is an analytic nondegenerate map, and $f \in C^{q}$. Let $H$ be an analytic submanifold of $U$ of codimension one, and $g$ be a metric on $U$ of class $C^{q-1}$ with $\left.g\right|_{H}$ $=F(f)$. We need that $q>9$ and is not equal to an integer. Let $\|v\|_{q}$ denote the $C^{q}$ norm in either $U$ or $H$ depending on the domain of the function $v$.

Theorem 4.1. There exists a constant $C$ depending on the values of $u$ in some complex ball of radius $r_{0}$ with the following property: If for some $r<r_{0}$ one has $\left\|f-\left.u\right|_{H}\right\|_{q}<C r^{9}$ and $\|g-F(u)\|_{q-1}<C r^{8}$, where the norms are over $H \cap \tilde{B}(r)$ and $\tilde{B}(r)$, then there exists a map $\tilde{u}: \tilde{B}\left(\frac{1}{2} r\right) \rightarrow E^{N}$ of class $C^{q-3}$ with $F(\tilde{u})=g$ and $\left.\tilde{u}\right|_{H}=f$.

Remarks. By $\left.\tilde{u}\right|_{H}=f$ we of course mean $H$ replaced by $H \cap \tilde{B}\left(\frac{1}{2} r\right)$. One can even find a $\tilde{u}$ with the additional property that $\tilde{u} \in C^{q^{\prime}-3}$ whenever, for some $q^{\prime}>q, f \in C^{q^{\prime}}$ and $g \in C^{q^{\prime}-1}$; here no additional estimates in $C^{q^{\prime}}$ are necessary. Thus the solution is roughly as smooth as the data. In particular, if $f$ and $g$ are of class $C^{\infty}$ and satisfy the hypotheses of the above theorem, then we can find a solution $\tilde{u} \in C^{\infty}$. 
Note we have not explicitly assumed our previous sufficient condition $\bar{L}>L$ or even the necessary condition $\bar{L} \geq L$. However, from the fact that $u: U \rightarrow$ $E^{N}$ is nondegenerate it follows that $\left.u\right|_{H}$ is also nondegenerate and satisfies $\bar{L}>L$, so that $\bar{L}>L$ holds for all $f$ near $\left.u\right|_{H}$.

Now we introduce new notation and modify old. For fixed $r$ let $\mathscr{D}^{i}=$ $\left\{z \in C^{n}|| \operatorname{Re} z\left|<r\left(\frac{1}{2}+2^{-i}\right),\right| I_{m} z \mid<2^{-i}\right\} ; \mathscr{D}^{\infty}=\left\{z \in \boldsymbol{R}^{n}|| z \mid<\frac{1}{2} r\right\}$. Let $|f|_{i}$ now denote the the sup norm in $\mathscr{D}^{i}$. Similarly define the norm on derivatives $|f|_{i, k}$. Let $\left\{f^{\nu}\right\}$ be a sequence converging pointwise to $f$ on $\mathscr{D}^{\infty}, f^{\nu}$ analytic on $\mathscr{D}^{\nu}$, with the property that for each $l$ with $f \in C^{l}\left(\mathscr{D}^{\infty}\right)$ there is a constant $C_{l}$ such that for all $\nu$

$$
\left|f^{\nu}-f^{\nu-1}\right|_{\nu} \leq C_{l} 2^{-\nu l}\left|f^{0}-f\right|
$$

This last norm is in $C^{l}(\tilde{B}(r))$. We denote such a convergence by $f^{\nu} \rightrightarrows f$. If $f^{\nu} \rightrightarrows f$ with respect to the fixed $r$, then we have the same type of convergence for all smaller values of $r$ too. See for instance [7] for a discussion of this convergence and a similar application. In particular, there are numbers $C_{l}$ depending only on $l, n$, and $N$ such that given $f$ and $f^{0}$ one can always find such a sequence $\left\{f^{\nu}\right\}$.

Basically one proves Theorem 4.1 as follows. First find some $v \in C^{q}(\tilde{B}(r))$ for which $\left.v\right|_{H}=f$ and $\|v-u\|_{q}$ is small. Now find $u_{i} \rightrightarrows v$ and $g_{i} \rightrightarrows g$ with $\left.g_{i}\right|_{H}=\left.F\left(u_{i}\right)\right|_{H}$ (take $u_{0}$ to be $\left.u\right)$. Consider the functional $\mathfrak{\Im}(v, g, w)=F(v+w)$ $-g . \mathfrak{F}$ is an analytic function of its arguments, and $\mathfrak{F}(u, F(u), 0)=0$. By Theorem $2^{\prime}$ of [6] we can, given $v$ and $g$, solve $\mathfrak{\Im}(v, g, w)=0$ for $w$ provided $\|v-u\|_{q}$ and $\|g-F(u)\|_{q-1}$ are small. We shall reprove this result in our present special case because, first, we must show $w=0$ on $H$ and must obtain precisely the statement of Theorem 4.1 and, second, the proof of Theorem 2' was omitted in [7]. But since the techniques have already appeared, we omit some details.

So we first construct the sequences $\left\{u_{i}\right\}$ and $\left\{g_{i}\right\}$. Assume $\left\|f-\left.u\right|_{H}\right\|_{q}<\varepsilon$. Set $v(x, y)=u(x, y)-u(x, 0)+f(x)$. So $\left.v\right|_{H}=f(x)$ and $\|v-u\|_{q}<\varepsilon$. Take any sequence $u_{i} \rightrightarrows v, u_{0}=u$. We now need a simple lemma.

Lemma. Consider a function $h(t, x), t \in \boldsymbol{R}^{a}, x \in \boldsymbol{R}^{b}$ for some integers $a$ and b. If $h^{i}(x) \rightrightarrows h(0, x)$, then there exists a sequence $\left\{h^{i}(t, x)\right\}$ with $h^{i}(t, x) \rightrightarrows$ $h(t, x)$ and $h^{i}(0, x)=h^{i}(x)$.

Take any sequence $\left\{\bar{h}^{i}\right\}, \bar{h}^{i}=\bar{h}^{i}(t, x)$, converging in the manner defined previously. It is obvious that $\left\{h^{i}\right\}$ defined by $h^{i}=h^{i}(t, x)=\bar{h}^{i}(t, x)-\bar{h}^{i}(0, x)$ $+h^{i}(x)$ has the desired properties. Applying this lemma to the relevant components of $g$ and using $\left.g\right|_{H}=F(f)=\left.F(v)\right|_{H}$ we find a sequence $\left\{g_{i}\right\}$, where $g_{i} \rightrightarrows g$ and $\left.g_{i}\right|_{H}=\left.F\left(u_{i}\right)\right|_{H}$, and also we take $g_{0}=F(u)$.

Consider the iteration

$$
w_{0}=0, \quad w_{n}=w_{n-1}-d F\left(u_{n-1}+w_{n-1}\right)^{-1}\left\{F\left(u_{n-1}+w_{n-1}\right)-g_{n-1}\right\} .
$$


If $w_{n}$ converges to some $w$, then clearly $F\left(u_{n}+w_{n}\right)$ converges to $g$, and so $F(v+w)=g$. Further if $\left.w_{n-1}\right|_{H}=0$, then also $\left.w_{n}\right|_{H}=0$ since $F\left(u_{n-1}\right)=$ $g_{n-1}$ as metrics restricted to $H$.

To begin the convergence proof let us recall that $\left|u_{i}-u_{i-1}\right|_{i} \leq$ $C_{q} 2^{-i q}\left\|v-u^{0}\right\|_{q}$ and $\left|g_{i}-g_{i-1}\right|_{i} \leq C_{q-1} 2^{-(q-1) i}\left\|F\left(u_{0}\right)-g\right\|_{q-1}$. By the Cauchy integral formula one has $|s(z)|_{i, k} \leq C 2^{i k} r^{-k}|s(z)|_{i-1}$. Now let $\left|\Delta h_{i}\right|=$ $\left|h_{i}-h_{i-1}\right|_{i}$ for any sequence $\left\{h^{i}\right\}$. Assume as an inductive hypothesis $\left|\Delta w_{i}\right| \leq$ $\lambda / 2^{i p}, i=1, \cdots, n-1$, for some $p$ and $\lambda$ to be chosen below (here $n$ is not related to the dimension of $U)$. For $i=1$ we have $\left|\Delta w_{1}\right|=\left|d F(u)^{-1}\left\{F(u)-g_{1}\right\}\right|_{\frac{1}{2} r}$ $\leq K\left|g_{0}-g_{\left.1\right|_{2} r, 2} \leq K(2 / r)^{2}\right| g_{0}-\left.g_{1}\right|_{r} \leq \lambda 2^{-p}$ if $|F(u)-g|_{p} \leq K r^{2} \lambda$. $K$, and later $\bar{K}_{1}$ and $K_{2}$, denote various constants which depend only on the values of $u$ in the complex ball of radius $r$. Note we have used the results of $\S 3$. Also, by Theorem 3.1, there is some $\varepsilon$ such that if $\left|u_{0}-\left(u_{n-1}+w_{n-1}\right)\right|_{n, 4}<\varepsilon$, then $d F^{-1}\left(u_{n-1}+w_{n-1}\right)$ can be defined and satisfies an estimate which in our present case yields $\left|\Delta w_{n}\right| \leq K\left|F\left(u_{n-1}+w_{n-1}\right)-g_{n-1}\right|_{n, 2}$.

Note that

$$
\left|u_{0}-\left(u_{n-1}+w_{n-1}\right)\right|_{n, 4} \leq \sum_{1}^{n-1}\left(\left|u_{i}-u_{i-1}\right|_{i+1,4}+\left|w_{i}-w_{i-1}\right|_{i+1,4}\right),
$$

and using Cauchy's estimate, our inductive estimate, and the fact that $u_{n} \rightrightarrows \vec{b}$ we obtain $\left|u_{0}-\left(u_{n-1}+w_{n-1}\right)\right|_{n, 4} \leq \varepsilon$ provided $p>4, q>4,\left\|u_{0}-v\right\|_{q} \leq$ $A r^{4} C_{q}\left(\frac{1}{2} \varepsilon\right)$, and $\lambda<\frac{1}{2} A \varepsilon r^{4}$ where $A$ depends only on $\min (q-4, p-4)$. Hence we think of $A$ as an absolute constant.

We now need to estimate $\left|\Delta w_{n}\right|$ using Theorem 3.1 to again bound our inverse operator. We have $F\left(u_{n-1}+w_{n-1}\right)=F\left(u_{n-2}+w_{n-2}\right)+d F\left(u_{n-2}+\right.$ $\left.w_{n-2}\right)\left(\Delta u_{n-1}+\Delta w_{n-1}\right)+F\left(\Delta u_{n-1}+\Delta w_{n-1}\right)$, and since $d F\left(u_{n-2}+w_{n-2}\right) \Delta w_{n-1}$ $=-F\left(u_{n-2}+w_{n-2}\right)+g_{n-2}$ one gets $\Delta w_{n}=d F^{-1}\left(u_{n-1}+w_{n-1}\right)\left\{d F\left(u_{n-2}+\right.\right.$ $\left.\left.w_{n-2}\right) \Delta u_{n-1}+F\left(\Delta u_{n-1}+\Delta w_{n-1}\right)+\Delta g_{n-1}\right\}$. So

$$
\begin{aligned}
\left|\Delta w_{n}\right| \leq & K\left\{\left|d F\left(u_{n-2}+w_{n-2}\right) \Delta u_{n-1}\right|_{n, 2}\right. \\
& \left.+\left|F\left(\Delta u_{n-1}+\Delta w_{n-1}\right)\right|_{n, 2}+\left|\Delta g_{n-1}\right|_{n, 2}\right\} \\
\leq & K K_{1}\left|\Delta u_{n-1}\right|_{n, 3}+K\left(\left|\Delta u_{n-1}\right|_{n, 3}+\left|\Delta w_{n-1}\right|_{n, 3}\right)^{2}+K\left|\Delta g_{n-1}\right|_{n, 2}
\end{aligned}
$$

and using the Cauchy estimate together with the inductive hypothesis one obtains

$$
\left|\Delta w_{n}\right| \leq K_{2}\left\{\left|u_{0}-v\right| r^{-3} \lambda^{-1}+\lambda 2^{-(p-6) n} r^{-6}+\left|g_{0}-g\right| r^{-2} \lambda^{-1}\right\} \lambda 2^{-p n}
$$

provided $q-3 \geq p \geq 6$ and $\left|u_{0}-v\right|<\lambda$. Now take $\lambda=\frac{1}{3} r^{6} K_{2}^{-1},\left\|u_{0}-v\right\|_{q}$ $\frac{1}{3} r^{9} K_{2}^{-1}$, and $\left\|g_{0}-g\right\|_{q-1}<\frac{1}{3} r^{8} K_{2}^{-1}$. We obtain $\left|\Delta w_{n}\right| \leq \lambda 2^{-p n}$, and by the induction this estimate is now valid for all $n$. Recall $\left|\Delta w_{n}\right|$ is an abbreviation for $\left|w_{n}-w_{n-1}\right|_{r 2-n}$. There thus is a function $w \in C^{p}$ with domain $\mathscr{D}^{\infty}$ such that $w_{n} \rightrightarrows w$. The convergence is now defined with respect to $\frac{1}{2} r$ rather than $r$. Also for $w$ to belong to $C^{p}$ one must assume $p$ is not an integer. Taking $p=$ 
$q-3$ proves the Theorem. It only remains to prove the remark after Theorem 4.1 , that is, to show we can even find one particular solution $\tilde{u}$ which is smooth as the data allow. To do this we would have to modify our iteration. Instead of using each $g_{n}$ in only one step of the iteration, solve the entire problem for $g$ replaced by (the analytic) $g_{n}$. Next consider the perturbation problem of going from the now known solution for $g_{n}$ to the desired solution for $g_{n+1}$. This allows one to keep better control on the rate of convergence.

\section{Main results}

We now come to our main result: every appropriate isometric embedding can be extended.

Theorem 5.1. Let $U$ be a Riemannian manifold, $H$ be a codimension one submanifold, $f: H \rightarrow E^{N}, N \geq \frac{1}{2} n(n+3)$, be a nondegerate isometric embedding, and $p$ be a point of $H$ at which $\bar{L}>L$. If $U, H$, and $f$ are of class $C^{\infty}$, then there exists a $C^{\infty}$ isometric embedding $\hat{f}$ of some open set containing $p$ with $\left.\hat{f}\right|_{H}=f$.

The following finite differentiability result also is valid.

Theorem 5.2. Let $g$ be a metric on $U, H$ a codimension one submanifold, $f: H \rightarrow E^{N}$ a nondegerate isometric embedding, and $p$ a point at which $\bar{L}>L$. If $H$ and $f$ are of class $C^{k}$, and $g$ of class $C^{k-1}$, where $k>17$ and is not an integer, then there exists an isometric embedding of a neighborhood of $p$, which is of class $C^{k-3}$ and extends $f$.

We prove this second theorem, and refer to [7] for the modifications necessary to get the $C^{\infty}$ result of Theorem 5.1. Also see the remarks at the end of the last section. Because of the local nature of this theorem we can think of $U$ as an open ball $\tilde{B}(r)$ in $\boldsymbol{R}^{n}$ and centered at the origin. Since $H$ is of class $C^{k}$, by means of a coordinate chart of class $C^{k}$ we can write $H=\left\{x=\left(x_{1}, \cdots\right.\right.$, $\left.\left.x_{n}\right) \mid x_{n}=0\right\}$. In these new coordinates, $f$ and $g$ remain of class $C^{k}$ and $C^{k-1}$, and $H$ is analytic. We arrange that the origin is the point $p$. With our usual convention on indices we have $g=g_{I J}$ and $\left.g\right|_{H}\left(x_{1}, \cdots, x_{n-1}\right)=g_{i j}\left(x_{1}, \cdots\right.$, $\left.x_{n-1}, 0\right)$. We shall now find an analytic $u^{0}: U \rightarrow E^{N}$ such that $F\left(u^{0}\right)$ and $\left.u^{0}\right|_{H}$ approximate $g$ and $f$ respectively, and then try to apply Theorem 4.1.

Let $\|v\|_{q, r}$ denote the norm in either $C^{q}(\tilde{B}(r))$ or $C^{q}(\tilde{B}(r) \cap H)$. By terminating the Taylor expansion of $f$ about the origin after $s$ terms we obtain an analytic map $f_{s}: H \rightarrow E^{N}$ with $\left\|f-f_{s}\right\|_{p, r} \leq C r^{s+1-p}$ for all real $p, 0 \leq p \leq s$. Similarly, we approximate $g_{I J}$ by $\left(g_{I J}\right)_{s-1}$, and obtain $\left\|g_{I J}-\left(g_{I J}\right)_{s-1}\right\|_{p-1, r} \leq$ $C r^{s+1-p}$. Consider the metric $\hat{g}$ given by $\hat{g}_{I n}=\left(g_{I n}\right)_{s-1}, \hat{g}_{i j}\left(y, x_{n}\right)=$ $\left(g_{i j}\right)_{s-1}\left(y, x_{n}\right)-\left(g_{i j}\right)_{s-1}(y, 0)+\left(F\left(f_{s}\right)_{i j}\right)(y)$ where $y=\left(x_{1}, \cdots, x_{n-1}\right)$, and note $F\left(f_{s}\right)=\left.\hat{g}\right|_{H}$ and $\|g-\hat{g}\|_{p-1, r} \leq C r^{s-p+1}$. From $\bar{L}>L$ at the origin and with respect to $f$ and $g$ we conclude the same for $f_{s}$ and $\hat{g}$. Thus by Theorem 1.1 we can find in a neighborhood of $p$ an analytic map $\hat{u}$ with $\left.\hat{u}\right|_{H}=f_{s}$ and $F(\hat{u})=\hat{g}$. Further since $N \geq \frac{1}{2} n(n+3)$, one can take $\hat{u}$ to be nondegenerate. Thus $\hat{u}$, 
or rather its complexification, is analytic and nondegenerate in some $B\left(r_{0}\right)$ in $C^{n}$. Since we have arranged that $H$ is also analytic, we can apply Theorem 4.1. Thus $\hat{u}$ determines a constant $C$ such that if for some $r<r_{0}$ and some $q>9$ we have $\left\|f-\left.\tilde{u}\right|_{H}\right\|_{q}<C r^{2}$ and $\|g-F(\hat{u})\|_{q-1}<C r^{8}$, then there exists some $\tilde{u} \epsilon$ $C^{q-3}\left(\tilde{B}\left(\frac{1}{2} r\right)\right)$ with $F(\tilde{u})=g$ and $\left.\tilde{u}\right|_{H}=f$. So we need $\left\|f-f_{s}\right\|_{q} \leq C r^{9}$ and $\| g-$ $\hat{g} \|_{q-1} \leq C r^{8}$. For $p=q$ and $s>q+8$ we have $\left\|f-f_{s}\right\|_{q} \leq C r^{9+\varepsilon}$ and $\| g-$ $\hat{g} \|_{q-1} \leq C r^{9+\varepsilon}$. So for $r$ sufficiently small and $q>9$ the conditions of Theorem 4.1 are satisfied. Thus there exists $\tilde{u} \in C^{q-3}$ with $\left.\tilde{u}\right|_{H}=f$ and $F(\tilde{u})=g$. We need $f$ and $g$ smooth enough to take $q+8$ derivatives, therefore in Theorem 5.2 we need $k>17$. As we have said, the proof of Theorem 5.1 is similar.

We can also use our knowledge of the linearized initial value problem to obtain results on isometric deformations. Let $U$ be an $n$-dimensional submanifold of $E^{N}$, and $H$ an $(n-1)$-dimensoinal submanifold of $U$. Does there exist an isometric deformation of $U$, which leaves $H$ unchanged as a submanifold of $E^{N}$ ? If $N \leq \frac{1}{2} n(n+1)$, then in general the answer is no. However for $N>$ $\frac{1}{2} n(n+3)$ and everything analytic, an affirmative answer was given in [8]. We want to now sketch a proof for the $C^{\infty}$ case.

By an isometric deformation of $u: U \rightarrow E^{N}$ we mean a family of embeddings $u_{t}: U \rightarrow E^{N}$ depending smoothly on $t$ with $u_{0}=u$ and $F\left(u_{t}\right)=F(u)$ for all $t$. In addition we want to require that $u_{t}$ equals $u$ on $H$.

Theorem. Let $U$ be a $C^{\infty}$ submanifold of $E^{N}, N \geq \frac{1}{2} n(n+3)+1$, which is nondegenerate at some point $p$, and $H$ be an arbitrary $C^{\infty}$ submanifold of $U$ also containing $p$. Then there exists a $C^{\infty}$ isometric deformation of some nighborhood $\tilde{U}$ of $p$, and this deformation leaves $\tilde{U} \cap H$ pointwise fixed.

Proof. Since $N>\frac{1}{2} n(n+3)$, we can find some $C^{\infty}$ vector $v$ which is orthogonal to $\mathcal{O}(U)$ at each point of $U$. Thus $d F(u) v=0$, and $v$ is an infinitesimal deformation. Take $v=0$ on $H$ but $v$ not identically zero near $p$. Now find two sequences $u^{\nu} \rightrightarrows u$ and $v^{\nu} \rightrightarrows v$ with the property that $d F\left(u^{\nu}\right) v^{\nu}=0$. Next find $g^{\nu} \rightrightarrows g=F(u)$ such that $\left.F\left(u^{\nu}+t v^{\nu}\right)\right|_{H}=\left.g^{\nu}\right|_{H}$ while at $t=0, g^{\nu}=$ $F\left(u^{\nu}\right)$ and $d g^{\nu} / d t=0$. Assume we have defined $0=w^{0}, w^{1}, w^{2}, \cdots, w^{\nu-1}$. Consider the iteration

$$
\begin{gathered}
w_{0}=w^{\nu-1}, \\
w_{n}=w_{n-1}-d F^{-1}\left(u^{\nu}+t v^{\nu}+w_{n-1}\right)\left\{F\left(u^{\nu}+t v^{\nu}+w_{n-1}\right)-g^{\nu}\right\} .
\end{gathered}
$$

If $w_{n}$ converges to some $w_{\infty}$, then $F\left(u^{\nu}+t v^{\nu}+w_{\infty}\right)=g^{\nu}$. Define $w^{\nu}$ by $w^{\nu}=w_{\infty}$. If also $w^{\nu}$ converges to some $w$, then $F(u+t v+w)=g$, and so we have an isometric deformation. This double iteration is one procedure for obtaining $C^{\infty}$ results; see [6] and the comments at the end of $\S 4$. It can be shown that for small values of $t$ the limit function $w$ does indeed exist: So it only remains to verify that $u(t)=u+t v+w$ leaves $H$ fixed and agrees with $u$ at $t=0$. Also we must show that $u(t)$ is not a trivial deformation; for perhaps $u(t)$ represents only a rotation of $E^{N}$. 
Assume that for some $\nu$ the map $w_{n-1}$ defined by our iteration is zero at $t=0$. Then the same is true for $w_{n}$ since $F\left(u^{\nu}\right)=g^{\nu}$. This shows $u(0)=u$. Similarly if $w_{n-1}=0$ on $H$, then we have the same for $w_{n}$ since $F\left(u^{\nu}+t v^{\nu}+\right.$ $\left.w_{n-1}\right)-g^{\nu}=0$ as a metric restricted to $H$. Thus $u(t)$ is an isometric deformation leaving each point of $H$ fixed. We now only must verify that $u(t)$ is not trivial. The only way $u(t)$ can be trivial is if at $t=0$ one has $d u / d t=A u$ for a constant anti-symmetric matrix A. But at $t=0, d w_{n} / d t=0$ (for the iteration starting at any $\nu$ ). This is proved by differentiating the equation which defines $w_{n}$ and using $F\left(u^{\nu}\right)=g^{\nu}$ and $d F\left(u^{\nu}\right) v^{\nu}=0$. One also needs the fact that $d F^{-1}$ has a Frechet derivative. So $d w^{\nu} / d t=0$ and $d u / d t=v$ at $t=0$. Could $v$ be of the form $v=A u$ ? Perhaps, but then just do the same iteration with $v$ replaced by $\Phi v$ for an arbitrary nonconstant function $\Phi$.

\section{References}

[1] C. Burstein, Ein Beitrag zum Problem der Einbettung der Riemannschen Räume in Euklideschen Räumen, Rec. Math. Moscow (Mat. Sb.) 38 (1931) 74-85.

[2] E. Cartan, Sur la possibilité de plonger un espace riemannien donné dans espace euclidean, Ann. Soc. Polon. Math. 6 (1927) 1-7.

[ 3 ] G. Darboux, Leçons sur la théorie générale des surfaces, Vol. 3, Gauthier-Villars, Paris, 1894.

[4] L. Eisenhart, Riemannian geometry, Princeton University Press, Princeton, 1949.

[ 5 ] A. Friedman, Local isometric embedding of Riemannian manifolds with identifinite metrics, J. Math. Mech. 10 (1961) 625-650.

[6] R. Greene, Isometric embeddings of Riemannian and pseudo-Riemannian manifolds, Amer. Math. Soc. Mem. No. 97, 1970.

[ 7 ] H. Jacobowitz, Implicit function theorems and isometric embeddings, Ann. of Math. 95 (1972) 191-225.

[ 8 ] — Deformations leaving a hypersurface fixed, Proc. Sympos. Pure Math. Vol. 23, Amer. Math. Soc., 1971, 343-351.

[9] M. Janet, Sur la possibilité de plonger un espace riemannien donné dans un espace euclidien, Ann. Soc. Polon. Math. 5 (1926) 38-43.

[10] K. Leichtweiss, Das Problem von Cauchy in der mehrdimensionalen Differentialgeometrie. I, Math. Ann. 130 (1956) 442-474.

[11] J. Nash, The imbedding problem for Riemannian manifolds, Ann. of Math. 63 (1956) 20-64.

[12] J. Weingarten, Über die Theorie der aufeinander abwickelbaren Oberflächen, Berlin, 1884 .

RICE UNIVERSITY 
